\title{
Histone deacetylase 3 is required for development and metamorphosis in the red flour beetle, Tribolium castaneum
}

\author{
Smitha George and Subba Reddy Palli ${ }^{*}$ (1)
}

\begin{abstract}
Background: Hormones are chemical communication signaling molecules released into the body fluids to stimulate target cells of multicellular organisms. We recently showed that histone deacetylase 1 (HDAC1) plays an important role in juvenile hormone (JH) suppression of metamorphosis in the red flour beetle, Tribolium castaneum. Here, we investigated the function of another class I HDAC member, HDAC3, and show that it is required for the normal development of $T$. castaneum.

Results: RNA interference-mediated knockdown of the HDAC3 gene affected development resulting in abnormally folded wings in pupae and adults. JH analog, hydroprene, suppressed the expression of HDAC3 in T. castaneum larvae. The knockdown of HDAC3 during the final instar larval stage resulted in an increase in the expression of genes coding for proteins involved in $\mathrm{JH}$ action. Sequencing of RNA isolated from larvae injected with dsRNA targeting malE (E. coli gene, control) or HDAC3 followed by differential gene expression analysis identified 148 and 741 differentially expressed genes based on the $P$-value $\leq 0.01$ and four-fold difference, and the P-value $\leq 0.05$ and two-fold difference, respectively. Several genes, including those coding for myosin-I heavy chain (Myosin 22), Shaven, and nuclear receptor corepressor 1 were identified as differentially expressed genes in HDAC3 knockdown larvae. An increase in histone $\mathrm{H3}$ acetylation, specifically H3K9, H3K18, and H3K27, was detected in HDAC3 knockdown insects.
\end{abstract}

Conclusion: Overall, these data suggest that HDAC3 affects the acetylation levels of histones and influences the expression of genes coding for proteins involved in the regulation of growth, development, and metamorphosis.

Keywords: HDAC3, Juvenile hormone, Tribolium castaneum, Acetylation, Histone H3

\section{Background}

Lysine acetylation is one of the major epigenetic modifications of proteins, which contributes to chromatin remodeling and expression of genes that regulate important biological processes [1]. In eukaryotes, the levels of acetylation of histones and other proteins are regulated by lysine acetyltransferases (KATs or Histone acetyltransferases HATs) and lysine deacetylases (KDACs or histone

\footnotetext{
* Correspondence: rpalli@uky.edu

Department of Entomology, University of Kentucky, Lexington, KY 40546, USA
}

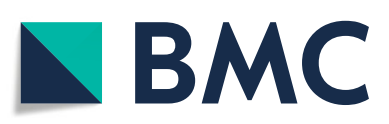

( The Author(s). 2020 Open Access This article is licensed under a Creative Commons Attribution 4.0 International License, which permits use, sharing, adaptation, distribution and reproduction in any medium or format, as long as you give appropriate credit to the original author(s) and the source, provide a link to the Creative Commons licence, and indicate if changes were made. The images or other third party material in this article are included in the article's Creative Commons licence, unless indicated otherwise in a credit line to the material. If material is not included in the article's Creative Commons licence and your intended use is not permitted by statutory regulation or exceeds the permitted use, you will need to obtain permission directly from the copyright holder. To view a copy of this licence, visit http://creativecommons.org/licenses/by/4.0/. The Creative Commons Public Domain Dedication waiver (http://creativecommons.org/publicdomain/zero/1.0/) applies to the data made available in this article, unless otherwise stated in a credit line to the data. moval of acetyl groups, respectively $[2,3]$. Lysine acetylation targets large macromolecular complexes responsible for various nuclear and cytoplasmic cellular processes: such as splicing, cell cycle, chromatin remodeling, DNA replication, etc. [4]. HDAC enzymes depend on zinc ions for their catalytic activity, and human HDACs were grouped into four classes $[5,6]$. Class I HDACs are localized in the nucleus, expressed universally, and play essential roles in cell proliferation, whereas class II and IV HDACs have a tissue-specific role $[7,8]$. 
Recent studies using HDAC inhibitors have suggested multiple roles for HDACs in cell proliferation, cell cycle arrest, and apoptosis [9]. The knockdown of $H D A C 3$ induced changes in gene expression, DNA damage, and caused cell cycle delay in mouse embryonic fibroblasts [10]. In Drosophila melanogaster, six HDACs (Rpd3, HDAC3, HDAC4, HDAC6-S, HDAC6-L, and Sir2) were characterized by studying temporal expression patterns and transcriptional profiling and the effect of HDAC inhibitors [11]. The D. melanogaster HDAC3 was cloned in 1998 and described as a metal-substituted enzyme [12]. RNA interference (RNAi)-mediated silencing of HDAC1 or HDAC3 in Drosophila S2 cells resulted in cell growth inhibition and deregulation of genes such as sox14, ecdysone-induced eip74ef, and $n v y$ [13]. Chemical genomics studies revealed that HDAC1, 2 and 3 are essential for core regulatory transcription and cell proliferation in cancer models [14]. Deacetylation by HDAC3 plays a vital role in the suppression of apoptosis in D. melanogaster imaginal tissue [15]. Acetylation of specific lysine residues of histones contributes to the dynamic regulation of ecdysone induced genes in D. melanogaster [16]. However, the role of acetylation in the regulation of juvenile hormone $(\mathrm{JH})$ action in insects is not well studied.

Juvenile hormones secreted by the corpora allata have multiple functions in an insect's life cycle and regulate diverse biological processes, including larval development, molting, metabolism, polyphenism, diapause, reproduction, and metamorphosis [17-21]. The JH signals are transduced through $\mathrm{JH}$ receptor, Methoprenetolerant (Met) [22, 23], Steroid receptor co-activator (SRC) [24], and CREB-binding protein (CBP) [25-27] (binding partners). $\mathrm{JH}$ represses the expression of genes involved in metamorphosis. $K r-h 1$ is an early $\mathrm{JH}$ response gene downstream of $\mathrm{Met}$, and RNAi mediated knockdown of Met or $\mathrm{Kr}-\mathrm{h} 1$ induces a precocious larvalpupal transition in the red flour beetle [28]. JH/Metdependent $K r$ - $h 1$ activity mediates the larval development. Lower JH titers result in lower levels of $K r-h 1$ expression in the last instar larvae allowing expression of pupal specifier, Broad complex and adult specifier, E93 and metamorphosis [29].

Recent research from our lab showed that the class I and II HDAC inhibitor Trichostatin A (TSA) mimics JH in the induction of $\mathrm{JH}$ response genes [27], suggesting a role for HDACs in JH action. We also demonstrated that HDAC1 influences $\mathrm{JH}$ action by regulating acetylation levels of histones, which promotes the expression of $\mathrm{JH}$ response genes [30]. In the present study, we focused on another member of the class I HDAC family, HDAC3 (TC006104). Knockdown of the HDAC3 gene during the final instar larval stage of the red flour beetle, Tribolium castaneum resulted in a pupa that showed abnormally folded wings and eventually died. RNA-seq analysis identified several genes including, Myo22, paired box protein Pax-5 (Shaven), and PDGF- and VEGF- related factor 3 (Pvf3), whose expression is influenced by HDAC3.

\section{Results \\ HDAC3 plays a key role in development and metamorphosis}

HDAC3 is a member of the Arginase/deacetylase superfamily that belongs to class I and is structurally and functionally related to HDAC1 and HDAC8 (Additional file 1, Fig. S1. A). Orthologues of HDAC3 are present in insects, other arthropods, and vertebrates (Additional file 1, Fig. S1. B, Gregoretti, Lee, and Goodson 2004). Injection of one microgram of dsRNA into newly molted last instar larvae induced 30\% larval mortality by eight days after dsRNA injection. The remaining larvae pupated but showed wing abnormalities, especially with wing folding, and could not complete development to the adult stage (Fig. 1Aa). Control larvae injected with dsmalE (dsRNA targeting malE gene from Escherichia coli) developed into normal pupae (Fig. 1Ab). Similarly, pupae with wing defects were observed when dsHDAC3 was injected into $72 \mathrm{~h}$-old (day 3) last instar larvae (Fig. 1Ac). Also, adults developed from pupae injected with dsHDAC3 showed wing defects (Fig. 1Ad). The pupae that developed from dsHDAC3 treated larvae are smaller in size than the control larvae treated with dsmalE (Additional file 1, Fig. S2). Conversely, dsmalE injected pupae developed into normal adults (Fig. 1Ae). Injection of dsHDAC3 into larvae, pupae and adults induced 78, 61 and $89 \%$ of knockdown of target gene respectively in larvae, pupae and adults (Fig. 1B) and resulted in 30, 41 and 54\% mortality, respectively in larvae, pupae and adults (Fig. 1C).

\section{Expression of $H D A C 3$ in larval and pupal stages}

Developmental expression of $H D A C 3$ during the penultimate and last instar larval and pupal stages was determined using reverse transcription-quantitative PCR (RTqPCR) and $H D A C 3$-specific primers (Additional file 1, Table S1). The HDAC3 mRNA levels were low during the penultimate and last instar larval and early pupal stages but increased at $24 \mathrm{~h}$ after pupal ecdysis. (Fig. 2A). The HDAC3 mRNA levels then decreased again, and lower levels were maintained throughout the pupal stage. In general, the HDAC3 mRNA levels were higher during the pupal stage when compared to those during the penultimate and last instar larval stages.

\section{$\mathrm{JH}$ analog hydroprene suppresses the expression of $H D A C 3$ in $T$. castaneum larvae}

The HDAC3 mRNA levels were significantly lower in hydroprene treated larvae when compared to those in 
A

$\mathbf{a}$

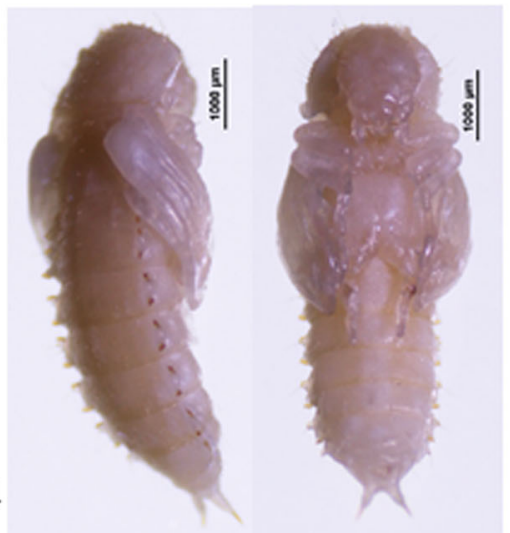

Injected at last larval stage $(0 \mathrm{~h})$

c

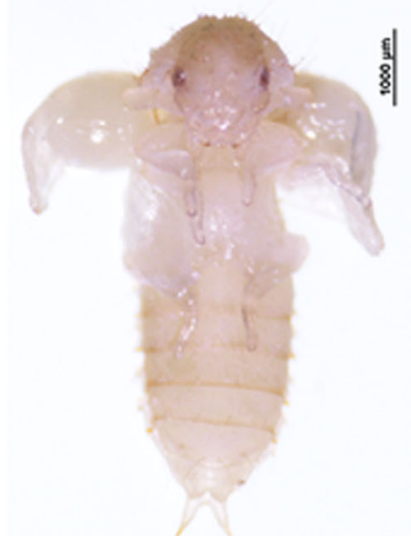

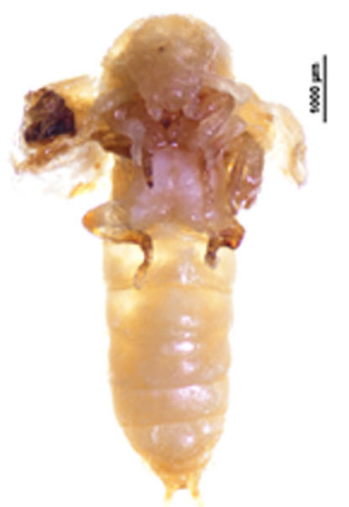

b

Control pupae (malE)

Injected after 3 days of Injected at pupal last larval stage $(72 \mathrm{~h}) \quad$ stage $(0 \mathrm{~h})$

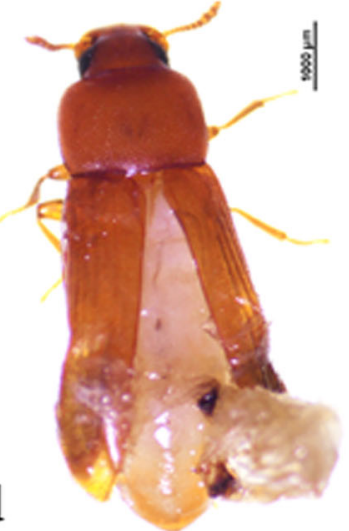

d

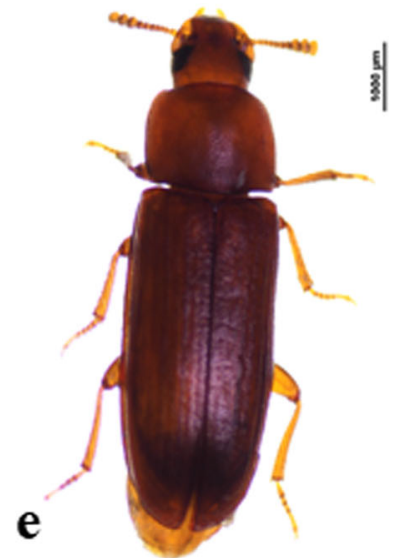

Control adult (malE)

B

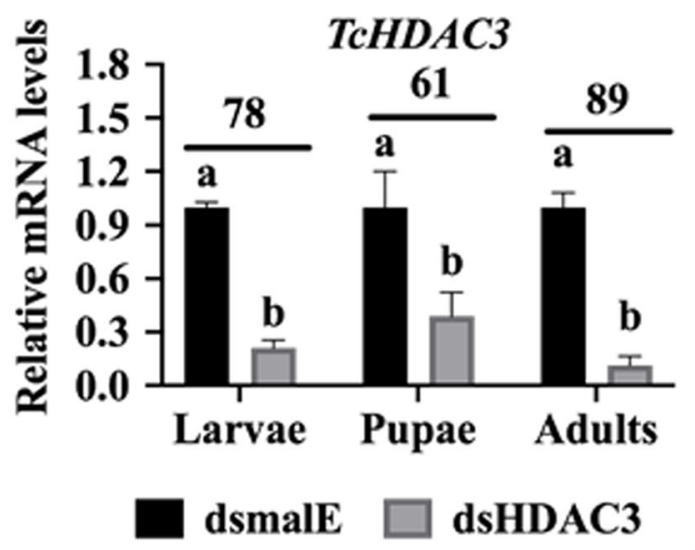

C

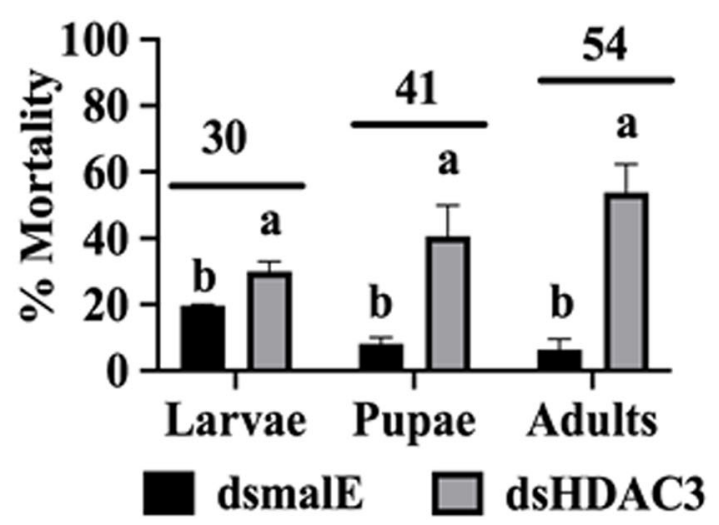


(See figure on previous page.)

Fig. 1 Phenotypes and mortality induced by RNAi-mediated knockdown of HDAC3 in T. castaneum. A a) dsHDAC3 was injected into the newly molted last instar larvae. Developmental defects and mortality were recorded every day until adult eclosion. The knockdown of the HDAC3 gene affected pupal development resulting in abnormally folded wings. b) Control larvae injected with dsmalE pupated in 5-6 days after injection and later emerged as healthy adults. c) The larvae injected with dsHDAC3 at $72 \mathrm{~h}$ after ecdysis to last instar larval stage pupated but showed abnormally folded wings. d) dsHDAC3 injected into newly formed pupae caused defects in the wing development. e) Healthy adults have emerged from the pupae injected with dsmalE. B HDAC3 mRNA levels were determined in larvae, pupae, and adults injected with dsHDAC3 or dsmalE. dsRNA were injected into day 0 last instar larvae, pupae and adults and the insects were collected on the third day after treatment, total RNA extracted and used to determine relative HDAC3 mRNA levels. Levels not connected by the same letter are significantly different. Mean \pm SE $(n=30)$ are shown. $C$ Injection of dsHDAC3 into day 0 last instar larvae, pupae and adults induced 30,41 and 54\% mortality, respectively mortality not connected by the same letter are significantly different. Mean $\pm S E(n=30)$ are shown

control larvae treated with solvent (Fig. 2B). As expected, the mRNA levels of $\mathrm{JH}$ response gene $K r$ - $h 1$ increased in hydroprene treated larvae when compared to those in control larvae treated with cyclohexane (Fig. 2B). Also, the difference in expression levels of HDAC3 in larvae, pupae and adults was detected (Additional file 1, Fig. S3). Higher HDAC3 mRNA levels were detected in wing discs when compared to the other tissues isolated from 72 -h-old last instar larvae (Additional file 1, Fig. S3). In contrast, no significant differences in HDAC3 mRNA levels were detected in different tissues dissected from pupae (Additional file 1, Fig. S3).

To determine whether the $\mathrm{JH}$ receptor, Methoprene tolerant, Met, mediates $\mathrm{JH}$ suppression of $H D A C 3$, we injected dsMet into last instar larvae and treated them with hydroprene or cyclohexane. As expected, the HDAC3 mRNA levels decreased in dsmalE (control) injected larvae treated with hydroprene but not in dsMet injected larvae treated with hydroprene (Fig. 2C). Also, $K r$ - $h 1$ mRNA levels increased in dsmalE (control) injected larvae treated with hydroprene but not in dsMet injected larvae treated with hydroprene (Fig. 2C). These data suggest that Met is required for JH III suppression of $H D A C 3$ gene expression.

\section{Knockdown of HDAC3 induces expression of genes involved in $\mathrm{JH}$ action and response in $T$. castaneum larvae and pupae}

$H D A C 3$ knockdown efficiency and its effect on the expression of $\mathrm{JH}$ response genes were tested using RTqPCR. A significant knockdown of $H D A C 3$ was detected in larvae collected at $12 \mathrm{~h}$ after dsHDAC3 injection (Fig. 3A). The Kr-h1, 4EBP, SRC, and CBP mRNA levels increased significantly in dsHDAC3 injected larvae when compared to those in dsmalE injected larvae. The expression of Met was not affected by HDAC3 knockdown. We also tested the housekeeping genes actin and heat shock protein (HSP90) to determine whether this effect is universal. Actin and HSP9O mRNA levels were not affected by $H D A C 3$ knockdown (Fig. 3A). A similar pattern of $H D A C 3$ knockdown and an increase in the expression of $K r-h 1,4 E B P$, and $S R C$ were detected in 24 h-old pupae developed from dsHDAC3 injected larvae
(Fig. 3B). The CBP mRNA levels did not increase in pupae developed from dsHDAC3 injected larvae. Also, the mRNA levels of the JH-response gene, G13402 did not increase in dsHDAC3 injected larvae (Fig. 3A) but increased in pupae developed from dsHDAC3 injected larvae (Fig. 3B).

To identify other target genes whose expression is affected by $H D A C 3$ knockdown, we sequenced the RNA isolated from dsHDAC3 and dsmalE injected larvae. Run summary and read count statistics of sequencing output are shown in Additional file 1, Table S2. The overall pattern of normalized mean expression values of differentially expressed genes (DEGs) is represented as a heatmap (Fig. 4A). The DEGs are shown as a volcano plot with red dots indicating statistically significant genes after the EDGE test between treatment and control (Fig. 4B). After statistical analysis using Baggerley's test to compare gene expression between dsHDAC3 and dsmalE treated insects, we identified 148 and 741 DGEs based on the $P$-value $\leq 0.01$ and four-fold difference, and the $P$-value $\leq 0.05$ and two-fold difference, respectively (Additional files 2 \& 3). Among these, 126 and 563 genes were up-regulated, and the rest of them were down-regulated under the two stringency conditions tested. Hormone response genes, $K r-h 1$, Ecdysone induced protein $78 \mathrm{C}$, and broad complex were upregulated in $H D A C 3$ knockdown larvae (Additional file 1, Table S3). Web-based GO analysis of differently expressed genes showed enrichment of GO terms for binding, especially nucleic acid and ion binding, regulation of the cellular process, biological regulation, and transport (Additional file 1, Fig. S4).

Twenty genes (Additional file 1, Table S4) that are upregulated in both $H D A C 3$ and $H D A C 1$ knockdown larvae [30] were selected for verification of RNA-seq data DEG predictions by RT-qPCR. The genes were selected based on the presence of a DNA-binding domain with possible functions as transcription factors, and RT-qPCR was used to determine their mRNA levels. Sixteen out of 20 genes tested showed an increase in their mRNA levels in $H D A C 3$ knockdown larvae when compared to those in control dsmalE treated larvae (Fig. 4C). Comparison of up-regulated genes between JH III [31] and dsHDAC3 
A
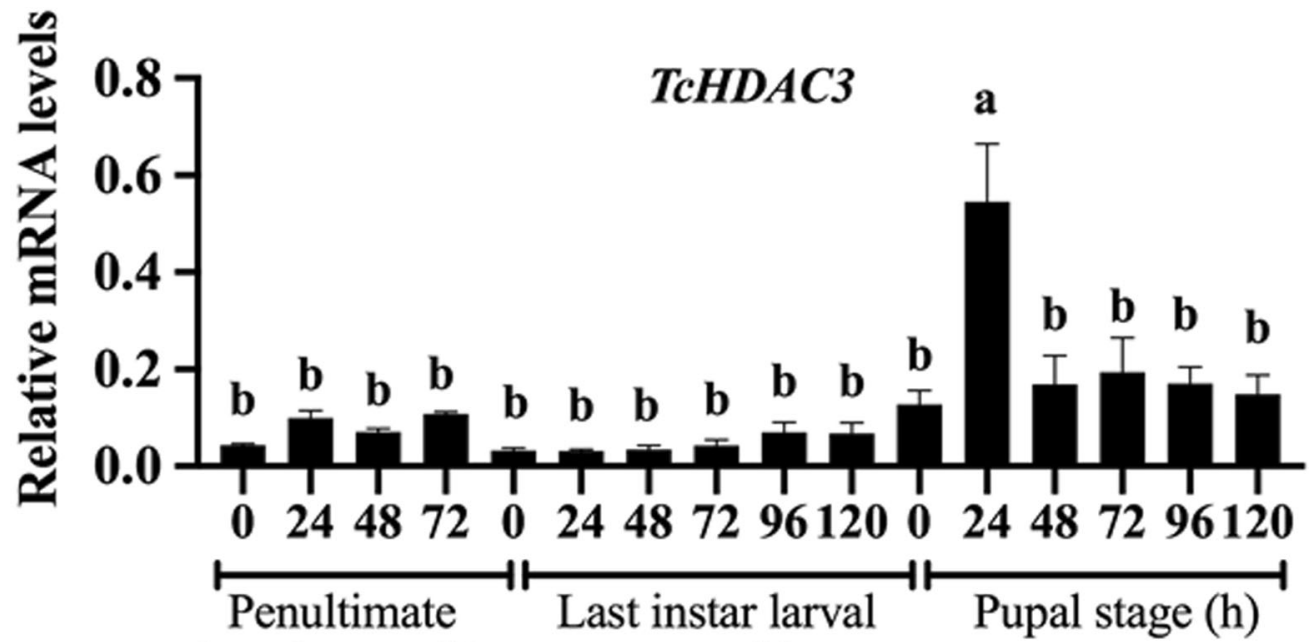
larval stage (h) stage (h)

B

$\left.\begin{array}{lll}\mathrm{C} & \mathbf{0 . 0 2 0}\end{array}\right] \stackrel{\text { a }}{\text { Met }}$
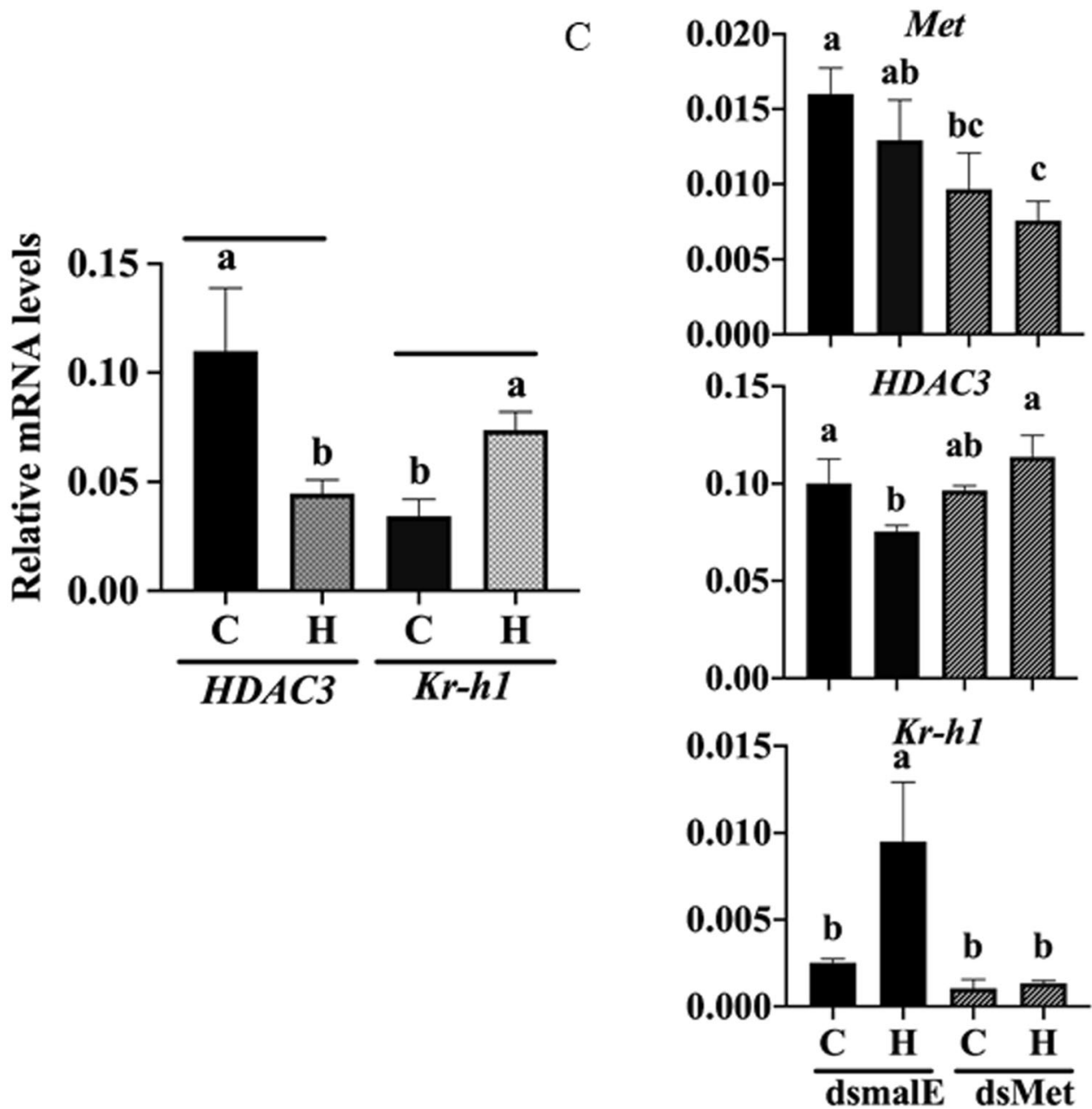

Fig. 2 (See legend on next page.) 
(See figure on previous page.)

Fig. 2 Developmental expression and JH induction of HDAC3 in T. castaneum. A HDAC3 mRNA levels were determined during the penultimate, last larval, and pupal stages at $24 \mathrm{~h}$ intervals. Total RNA isolated from a pool of two larvae for each replication was converted to cDNA and used in RT-qPCR to determine the relative HDAC3 mRNA levels. Mean \pm SE $(n=4)$ are shown. Levels not connected by the same letter are significantly different. B JH suppresses the expression of HDAC3 in T. castaneum larvae. S-Hydroprene $(\mathrm{H}, \mathrm{JH}$ analog) was dissolved in cyclohexane (C) and topically applied to $48 \mathrm{~h}$-old last instar larvae $(0.5 \mu \mathrm{L}$ of $2 \mu \mathrm{g} / \mu \mathrm{L})$. At six hours after treatment, total RNA was isolated and subjected to RT- $\mathrm{qPCR}$. The expression of the JH response gene $\mathrm{Kr}-\mathrm{h} 1$ was significantly induced, and HDAC3 was significantly suppressed, mean $\pm \mathrm{SE}(n=4)$ are shown. Levels not connected by the same letter are significantly different. C Met is required for suppression of HDAC3 by hydroprene. Newly molted last instar larvae were injected with dsMet or dsmalE. At $48 \mathrm{~h}$ after injection of dsRNA, the larvae were treated with hydroprene. Total RNA isolated from larvae was converted to CDNA and used to quantify $K r-h 1, H D A C 3$ and, Met mRNA levels. The data shown are mean \pm SE $(n=4)$. The data were analyzed using analysis of variance, each pair student's $t$-test. Mean values with the same letter are not significantly different from each other. C, cyclohexane; H, hydroprene

treated larvae identified six common genes, including $K r$-h1 (Additional file 1, Table S5). Six genes that code for proteins containing zinc finger COG5048 domains found in $K r$-h1 were also up-regulated in $H D A C 3$ knockdown larvae (Additional file 1, Table S6).

\section{Identification of genes affected by both HDAC3 knockdown and TSA treatment}

TSA selectively inhibits class I and II HDACs and was shown to alter gene expression by preventing the removal of acetyl groups from histones [32]. Previous studies from our lab identified TSA induced genes in T. castaneum TcA cells [31]. Comparison of TSA induced genes with up-regulated genes in $H D A C 3$ knockdown insects identified multiple genes (5.3\% of DGEs) that are common in both the treatments (Additional file 1, Fig. S5). The common genes identified from this analysis are listed in Additional file 4 . To verify the results, we selected nine genes from this list (Additional file 1, Table S7) and determined their mRNA levels in dsHDAC3 treated $T$. castaneum pupae (Fig. 5A) and TcA cells (Fig. 5B). Myo22 (myosin-I heavy chain/TC008923), shaven (paired box protein Pax5/TC003570) and Pvf3 (PDGF- and VEGF-related factor 3/TC008417) were significantly up-regulated in dsHDAC3 treated T. castaneum pupae and TcA cells when compared to their expression in control insects and cells treated with dsmalE (Fig. 5A, B). We also confirmed the significantly higher levels of neprilysin-11 (TC013029) in pupae treated with dsHDAC3 when compared to that in control pupae treated with dsmalE (Fig. 5A). Also, zinc finger protein 2-like (TC032605) and muscle M-line assembly protein unc-89 (TC003005) were significantly upregulated in TcA cells treated with dsHDAC3 (Fig. 5B). Since HDAC3 deacetylates co-activators like acetyltransferases p300/CBP, p300/CBP-associated factor (PCAF) $[33,34]$, we compared lists of TSA induced genes, upregulated genes in $H D A C 3$ knockdown insects and downregulated genes from CBP knockdown cells [26]. Common genes identified from this comparison are listed in Additional file 1, Table S8.

\section{HDAC3 regulates acetylation levels of histone $\mathrm{H} 3$}

Total proteins were isolated from the dsHDAC3-treated last instar larval tissues and subjected to the western blot assay using acetyl-histone $\mathrm{H} 3$ antibody sampler kit \#9927 (Cell Signaling, MA) to determine the targets of $H D A C 3$ deacetylation. We evaluated the various lysine acetylation sites of histone H3 using Lys9, Lys14, Lys18, Lys27, and Lys56 specific antibodies. Increased acetylation of H3K9 and H3K27 was detected in dsHDAC1, and dsHDAC3 treated larvae compared to their levels in dsmalE treated larvae (Fig. 6A, B). These data suggest that $\mathrm{H} 3$ is one of the targets for HDAC1 and HDAC3.

\section{Discussion}

Recent research in our laboratory demonstrated that HDAC1 suppresses $K r-h 1$ gene expression and regulate $\mathrm{JH}$ suppression of metamorphosis in T. castaneum [30]. In the current studies, we investigated the role of the other member of the HDAC class I, the HDAC3. Unlike HDAC1 knockdown, which causes complete lethality during the larval stage, some of the $H D A C 3$ knockdown larvae undergo pupation, but the pupae exhibited defects, especially wing folding and the pupae that developed from dsHDAC3 treated larvae are smaller in size compared to the control larvae treated with dsmalE (Additional file 1, Fig. S2). Injection of dsHDAC1 into $T$, castaneum induced a block in growth and development and $100 \%$ mortality of larvae before pupation [30].

In contrast, HDAC3 knockdown is less severe, and some of the treated larvae completed larval development and died during the pupal stage. Some of the differences may be due to differences in the expression pattern of these two HDACs during the last larval stage. Further research is needed to uncover differences in the function of these two Class 1 HDACs. In D. melanogaster, mutations in $H D A C 3$ caused death during the late third instar larval and early pupal stages. Also, the imaginal discs are significantly reduced, and the pouch region of the wing disc was smaller in size compared to the wild-type [15]. RNAimediated HDAC3 knockdown in the beetle, Gnatocerus cornutus, caused a reduction in hind wing size [35]. 


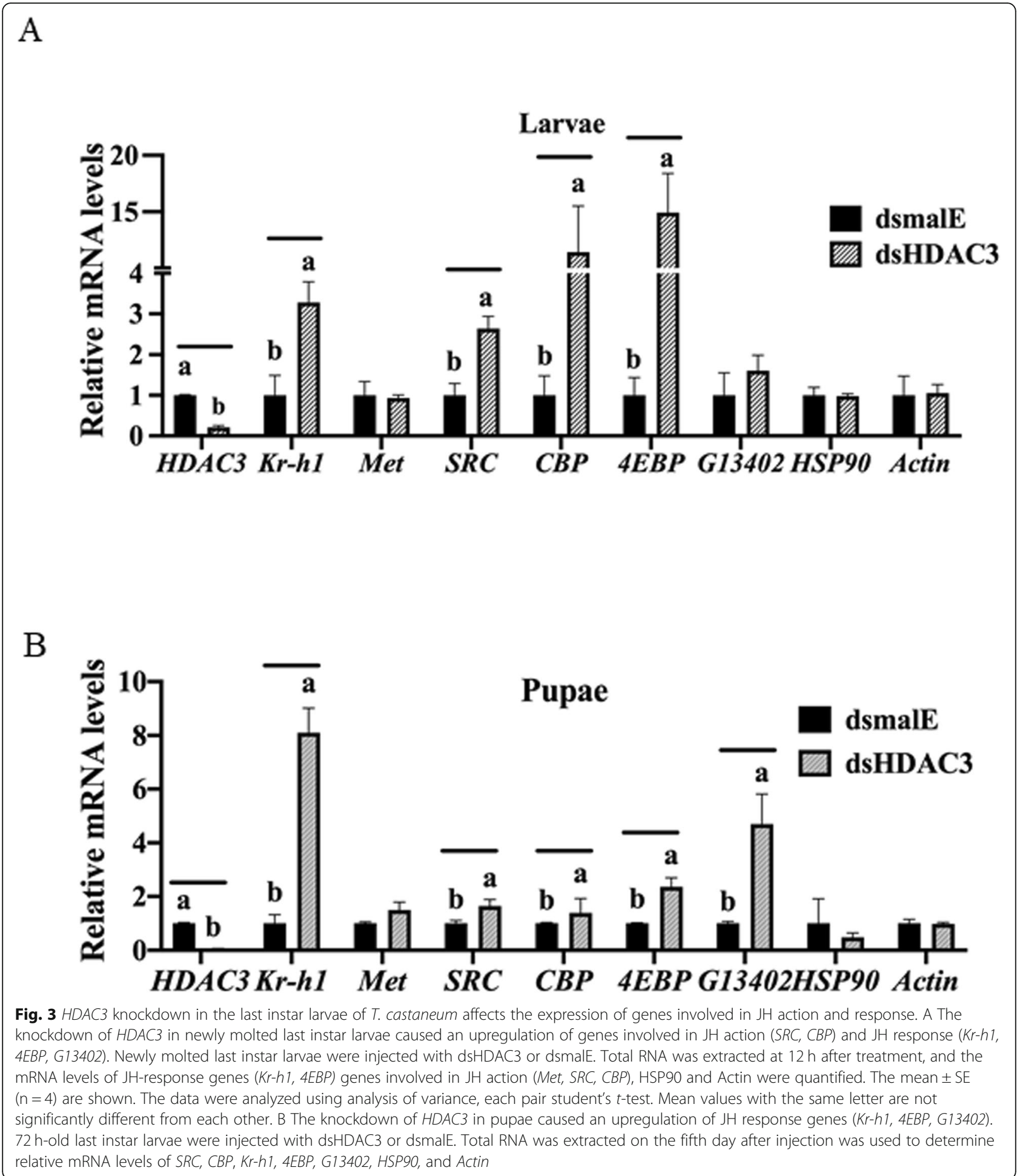

One of the primary outcomes of this research is the discovery that HDAC3 is required for normal larval, pupal and adult development in $T$. castaneum. The knockdown of $H D A C 3$ in newly molted last instar larvae caused an upregulation of genes involved in $\mathrm{JH}$ action $(S R C, C B P)$ and $\mathrm{JH}$ response $(K r-h 1$ and $4 E B P)$. In $D$. melanogaster, HDAC3 plays a crucial role in development, consistent with their relatively high expression during the embryonic and adult stages [11]. Our developmental expression studies showed a significant upregulation of the $H D A C 3$ gene expression in $24 \mathrm{~h}$-old pupae (Fig. 2A). Previous studies reported that T. castaneum 


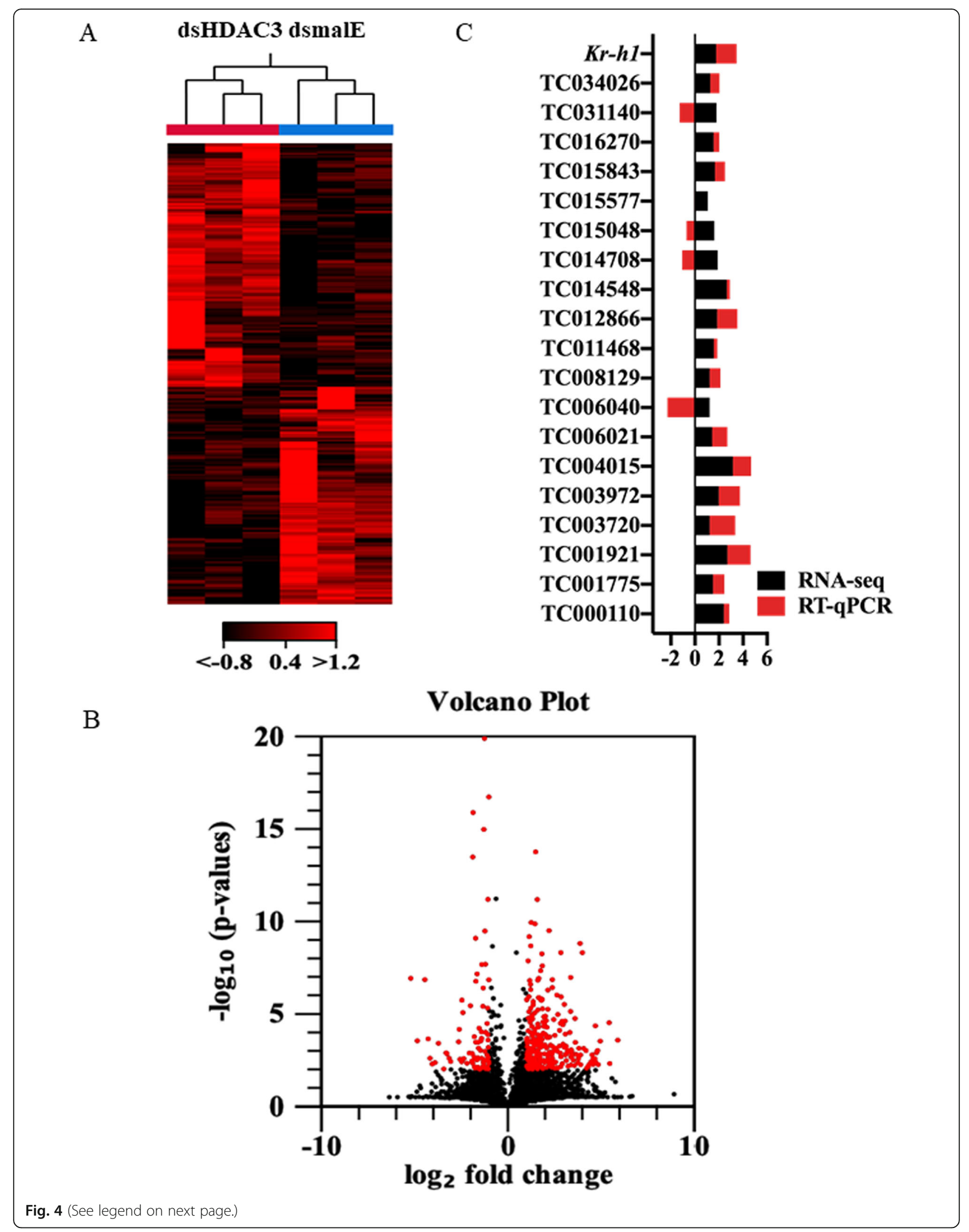


(See figure on previous page.)

Fig. 4 HDAC3 knockdown in the last instar larvae of T. castaneum affects the transcription of genes involved in multiple pathways. A Heatmap of RNA-seq data. The heatmap of normalized mean expression values of 741 differentially expressed genes $(\geq 2$ - fold and a $P<0.05$ ) between dsmalE and dsHDAC3 treated insects. B Differentially expressed genes identified after HDAC3 knockdown represented as the volcano plot. The $X$ and $Y$-axis represent the $-\log _{10} P$-values and $\log _{2}$ fold change of mean normalized values, respectively. The red dots indicate the genes that showed $\geq 2$ - fold difference in expression with a $P<0.05$. C RT-qPCR verified the expression of 20 selected genes from the up-regulated group (RNA-seq data). Descriptions of genes are listed in (Additional file 1, Table S4)

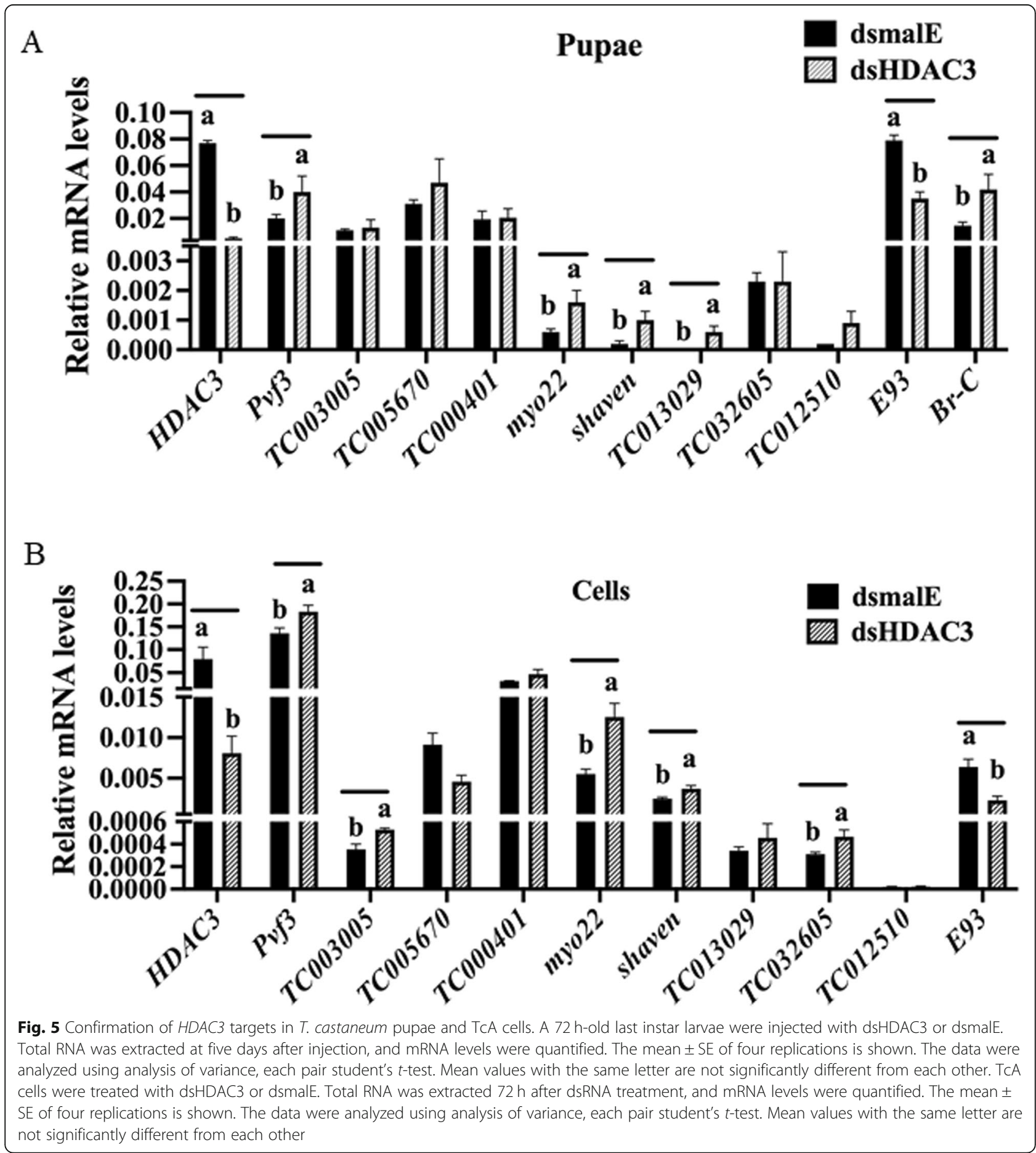




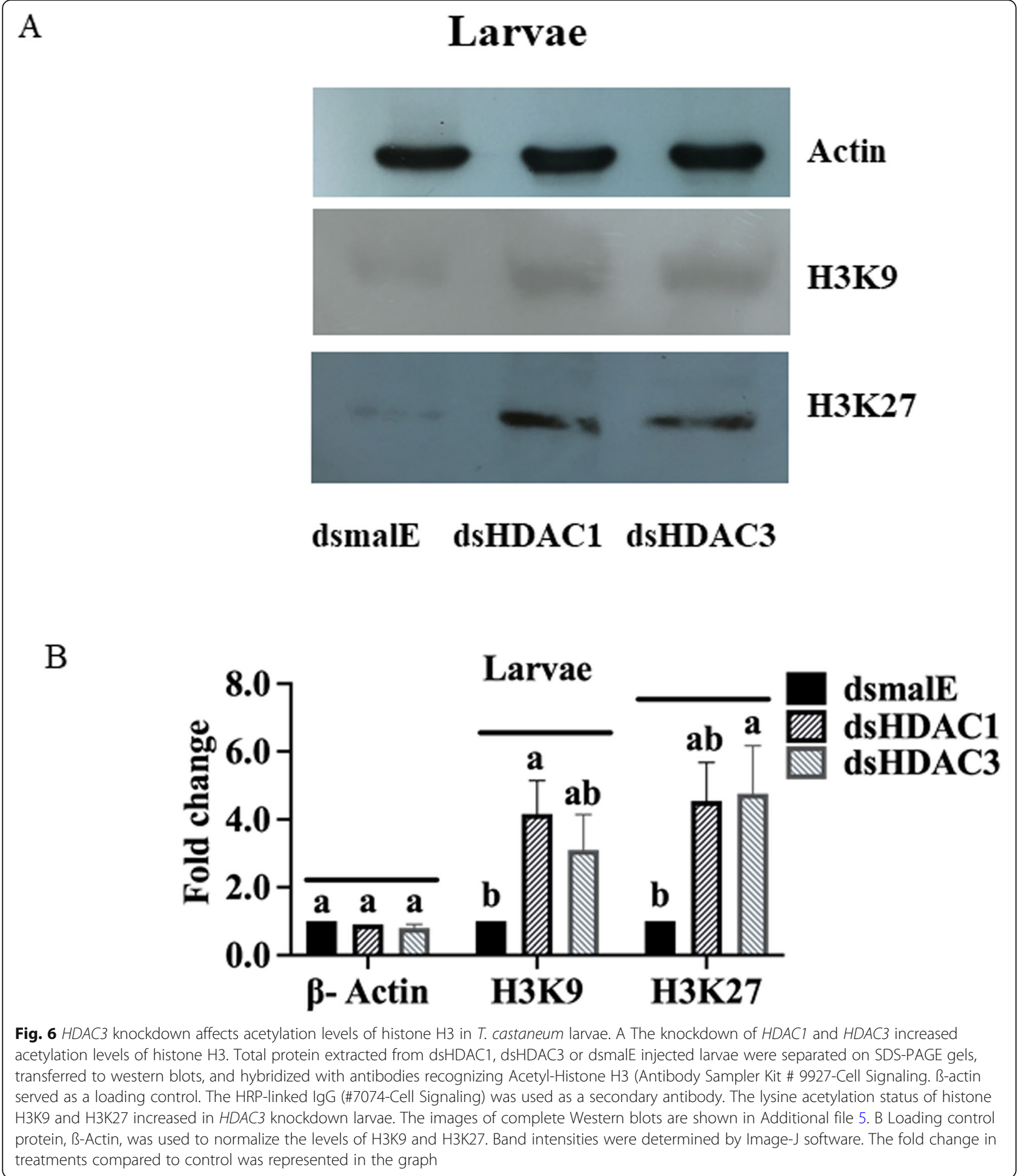

HDAC3 is expressed during all developmental stages, and the highest mRNA levels were detected in one-dayold pupae [36]. We also demonstrated that the depletion of HDAC3 during the pre-pupal stage (72-h old) T. castaneum results in abnormal pupal development. Interestingly, transcription factor E93 (adult specifier) was down-regulated in dsHDAC3 knockdown pupal and cell samples (Fig. 5A, B). In T. castaneum and D. melanogaster, E93 promotes adult metamorphosis and represses the expression of antimetamorphic genes $K r-h 1$ and pupal specifier, Broad-complex [37]. In HDAC3 knockdown insects, we found up-regulation of $K r-h 1, B r-C$, 
and downregulation of E93. The timing and expression levels of $\mathrm{Kr}-\mathrm{hl}, \mathrm{Br}-\mathrm{C}$, and $E 93$ (metamorphic genetic network) facilitate the proper larval-pupal-adult transition in holometabolous insects [38]. Based on these data, we propose that the misregulation of critical hormonerelated genes caused the abnormal pupal development in $H D A C 3$ knockdown insects. In general, histone acetylation and deacetylation at the promoter region are associated with transcription activation and repression, respectively [39]. Interestingly, differential gene expression analysis of sequences of RNA isolated from dsHDAC3 and dsmale treated larvae identified 563 (76\%) up-regulated, and 178 (24\%) down-regulated genes. The data suggest that the HDAC3 is involved in suppression of gene expression in T. castaneum larvae. The maintenance of equilibrium between acetylation and deacetylation of histones and non-histone proteins is essential for healthy cell growth. Loss of HDAC1 or HDAC3 leads to cell growth inhibition and overexpression of genes involved in lipid metabolism, DNA replication, cell cycle regulation, and signal transduction [13]. Histone acetyltransferases and deacetylases control cell proliferation and differentiation [40].

Myo22, a myosin heavy chain gene essential for muscle development, is a common gene up-regulated by HDAC3 knockdown larvae, and TSA treated cells. (Additional file 4, Fig. 5A, B). Functions of myosin heavy chain (MHC) family genes in $T$. castaneum were reported recently [41]. The TcMyo20 is essential for wing and leg morphogenesis in T. castaneum. MHC isoforms regulate muscle function and are critical for specialized functions such as flying and jumping in D. melanogaster [42]. The activity of HDACs (class I, II) promoted swimming performance, but reduced slow and fast MHC content in cardiac and skeletal muscles in zebrafish [43] Trichostatin A (TSA, Class I, II HDAC inhibitor) induces $\mathrm{JH}$ response genes in a dose-dependent manner in T. castaneum [27]. TSA works as an epigenetic modulator, and deacetylation by TSA regulates the expression of key players involved in $\mathrm{JH}$ and 20E action in TcA cells [31]. HDAC inhibition by TSA led to an increase in the concentration of MHC in both skeletal and cardiac muscle in zebrafish (Danio rerio) [43]. Histone acetyltransferases $\mathrm{CBP} / \mathrm{p} 300$ binds to the muscle-specific promoter, and this leads to enhanced transcription of muscle-specific genes [40]. Our data suggest that HDAC3 could play an important role in muscle development and function. However, further studies are needed to identify the exact mechanism of HDAC3 suppression of Myo22 in T. castaneum.

Our results also demonstrated the role of HDAC3 in the regulation of 'shaven,' a transcription factor that is involved in the development of a variety of sensory organs [44]. Additionally, we confirmed the upregulation of Pvf3 in HDAC3 knockdown larvae. Pvf3 functions in embryonic plasmatocyte survival and migration in $D$. melanogaster [45]. Interestingly, LOC103313779 (nuclear receptor corepressor 1, TC006021; Drosophila orthologue for smrter (smr), corepressor of ecdysone receptor) was up-regulated in $H D A C 3$ knockdown samples (Additional file 1, Table S3). Suppression of HDAC3 enhances apoptosis induced by paclitaxel in human maxillary cancer cells [46].

Histone deacetylase 3 is associated with the nuclear receptor corepressor complex containing $\mathrm{N}-\mathrm{CoR}$ and SMRT (Silencing mediator for retinoid and thyroid hormone receptors) [47, 48]. HDAC3 is crucial for repression by multiple nuclear receptors, and the N-CoRHDAC3 complex plays a unique role in thyroid hormone receptor-mediated gene repression in human 232 $T$ cells [49]. Our previous studies demonstrated that the HDAC1/SIN3 multiprotein complex regulates the expression of $K r-h 1$ [30]. Further studies with the NCoRHDAC3 complex is required to identify the mechanism of gene regulation by HDAC3.

In $D$. melanogaster, knockdown of RPD3 by RNAi affects global histone acetylation, especially K9/14 of histone H3 and K8/K12 of histone H4 [50]. Similarly, HDAC1 and RPD3 disruptions result in histone $\mathrm{H} 4$ and $\mathrm{H} 3$ hyperacetylation, especially at $\mathrm{H} 3 \mathrm{~K} 9 / 18$ and $\mathrm{H} 4 \mathrm{~K} 5$ and $\mathrm{K} 12$ in the Saccharomyces cerevisiae [51]. Our western blot results showed that RNAi- mediated knockdown of HDAC3 results in an increase in acetylation of Histone H3K9 and H3K27 in T. castaneum larvae (Fig. 6). In rats, acetylation of H3K9 increased in cortical neurons accompanied by a reduction of nuclear localization of HDAC3 [52]. Treatment of HeLa cells with TSA or silencing of $H D A C 3$ expression by small interfering RNA caused hyperacetylation of Lys-9 in histone $\mathrm{H} 3$ near the growthdifferentiation factor 11 (gdf11) promoter [53]. HDAC3 selectively represses CREB3-mediated transcription and migration of metastatic breast cancer cells [54]. The histone $\mathrm{H} 3$ acetylase dGcn 5 is a notable player in D. melanogaster metamorphosis [55]. HDAC3 deacetylases myocyte enhance factor 2, a transcription factor essential for controlling gene expression, muscle differentiation, apoptosis, and survival of different cell types [33]. These data suggest that $H D A C 3$ suppression induces the acetylation status of histone H3. WEGO plot showed enrichment of terms involved in biological regulation, regulation of the cellular process, signal transduction, ion binding, and catalytic activity in $H D A C 3$ knockdown insects.

\section{Conclusions}

$H D A C 3$ knockdown interferes with the $\mathrm{JH}$ response gene $K r$ - $h 1$, pupal specifier $B r-C$, and the adult specifier transcription factor $E 93 . \mathrm{JH}$ response gene $\mathrm{Kr}-\mathrm{h} 1$ and $\mathrm{Br}-\mathrm{C}$ was significantly up-regulated by $H D A C 3$ 
knockdown; the ecdysone response gene E93 was significantly down-regulated. Six genes were commonly upregulated in dsHDAC3- and TSA-treatment, and downregulated in dsCBP treatment (Additional file 1, Table S8). We further confirmed our result with RT-qPCR and identified that TC003570 (Shaven) and TC008417 (pvf3), which are important for sensory and cell proliferation, respectively, are significantly up-regulated in $H D A C 3$ knockdown pupae. A model for the HDAC3 function is shown in Additional file 1, Fig. S6. In conclusion, we identified that HDAC3 reduction in T. castaneum affects the genes responsible for muscle development and signal transduction, and thereby affecting the development and metamorphosis.

\section{Methods}

\section{Insect rearing and cell culture}

T. castaneum GA-1 strain [56] beetles were reared on organic wheat flour (Heartland Mill, Marienthal, KS) containing $10 \%$ dried baker yeast (MP biomedicals, Solon, $\mathrm{OH})$ at $30{ }^{\circ} \mathrm{C}$ and $65 \pm 5 \%$ relative humidity. The T. castaneum cells (BCIRL-TcA-CLG1, TcA) were grown in EX-CELL 420 (Sigma-Aldrich, St-Louis, MO) medium supplemented with 10\% Fetal Bovine Serum (FBS, VWR-Seradigm, Radnor, PA) at $28^{\circ} \mathrm{C}$ [57].

\section{Hormone treatments}

Insect growth regulator/JH analog, S-Hydroprene (Sigma-Aldrich, MO), was used to test the response of the HDAC3 gene to JH. Cyclohexane was used as a solvent control where hydroprene was dissolved to obtain the concentration of $2 \mu \mathrm{g} / \mu \mathrm{l}$, and $1 \mu \mathrm{g}(0.5 \mu \mathrm{l} /$ larvae $)$ hydroprene was topically applied to $48 \mathrm{~h}$-old final instar larvae for in vivo hormone treatments. Six hours after treatment, the samples were collected and analyzed by RT-qPCR. The relative HDAC3 and Kr-h1 mRNA were determined.

\section{Double-stranded RNA synthesis (dsRNA) treatment and differential gene expression analysis}

RNA isolation, cDNA synthesis, quantitative reverse transcription PCR (RT-qPCR), Double-stranded RNA synthesis (dsRNA) and microinjection, RNA-sequencing (RNA-seq) and data analysis and annotations were performed as described our previous publications [26, 30, 31]. RT-qPCR was performed using gene-specific primers (Additional file 1, Table S1) and iTaq Universal SYBR Green Supermix (BioRad, Hercules, CA) in Applied Biosystems StepOnePlus Real-time PCR instrument. The libraries for RNA sequencing were prepared, as described previously [26]. The pooled libraries were sequenced using the Illumina Hiseq 4000 sequencer at Duke University Sequencing and Genomic Technologies (NC, USA). The raw reads were demultiplexed, trimmed, and transcripts were mapped back to the $T$. castaneum reference genome (assembly Tcas5.2) using the CLC genomic workbench pipeline (Version 11.0.1).

\section{Protein extraction and western blotting}

The larvae were cleaned with ice-cold PBS and lysed in RIPA lysis buffer (Radioimmunoprecipitation assay buffer- $0.5 \mathrm{M}$ Tris- $\mathrm{HCl}, \mathrm{pH} 7.4,1.5 \mathrm{M} \mathrm{NaCl}, 2.5 \%$ deoxycholic acid, 10\% NP-40, 10 mM EDTA- Millipore Sigma, MA, USA) containing Halt protease inhibitor cocktail (ThermoFisher, Rockford, IL, USA). The lysate was clarified in a microfuge at $8000 \mathrm{rpm}$ for $5 \mathrm{~min}$. The proteins in the supernatant were precipitated by adding in order 1:8:1 ratio of lysate: ice-cold acetone: Trichloroacetic acid (TCA), and incubating at $-20^{\circ} \mathrm{C}$ overnight. The solution was then centrifuged in a microfuge at $12000 \mathrm{rpm}$ for $20 \mathrm{~min}$. The pellet was washed in ice-cold acetone twice to remove the TCA traces, air-dried, and dissolved in $10 \%$ SDS. The proteins quantified using Bio-Rad protein assay concentrate and standard. Samples were denatured $\left(95-100{ }^{\circ} \mathrm{C}\right.$ for $\left.5 \mathrm{~min}\right)$ in $5 \mathrm{X}$ SDS loading buffer to denature and stored at $-20^{\circ} \mathrm{C}$ for future use. An equal amount of proteins were loaded and resolved on $12 \%$ SDS-polyacrylamide gels, along with the protein ladder. The proteins were transferred from the gel to the polyvinylidene difluoride (PVDF) membranes (Bio-Rad, Hercules, CA) and blocked with 5\% nonfat dried milk blocking buffer for one hour at room temperature. The membranes were washed and incubated with $1000 x$ diluted primary lysine-acetylated antibody-Histone H3 antibody sampler kit \#9927 (Cell Signaling Technology, Danvers, MA) overnight at $4{ }^{\circ} \mathrm{C}$ with gentle shaking. The membranes were washed three times in Tris-buffered saline with Tween 20 (TBST) and incubated with 1000x diluted HRP-conjugated secondary antibody (\#7074- Cell Signaling) in blocking buffer at room temperature for one hour as described previously [58]. The signals were developed with Supersignal West Femto Maximum sensitivity Substrate (ThermoFisher, IL), following the manufacturer's protocol. The images were acquired in a darkroom using the chemiluminescence technique. Western blot bands were quantified by Image-J software and normalized with $ß$-Actin loading control protein.

\section{Statistical analysis}

JMP Pro 14.0 (SAS Institute Inc., Cary, NC) software was used for $t$-test, comparing means, $P$-value $<0.05$.

\section{Supplementary information}

Supplementary information accompanies this paper at https://doi.org/10 1186/s12864-020-06840-3.

\section{Additional file 1.}

Additional file 2.

Additional file 3 . 


\section{Additional file 4}

Additional file 5.

\section{Abbreviations}

HDAC3: Histone deacetylase 3; JH: Juvenile hormone; SRC: Steroid receptor Co-activator; CBP: CREB-binding protein; TSA: Trichostatin A; 4EBP: Eukaryotic translation initiation factor 4E-binding protein 2; E93: Transcription factor E93; Kr-h1: Krüppel homolog 1; 20E: 20-hydroxyecdysone; DEG: Differentially expressed gene; dsRNA: Double-stranded RNA

\section{Acknowledgments}

We thank Dr. Jingjing Xu for help with initial work on the project. Dr. Manohar Chakrabarti (Hunt lab) for help with RNA-seq analysis and Yaoyu Jiao for Tribolium castaneum culture maintenance.

\section{Authors' contributions}

SG and SRP designed the research methodology. SG conducted experiments and formal analysis. SG and SRP wrote and edited the manuscript. All authors read and approved the final manuscript.

\section{Funding}

This work was supported by grants from the National Institute of Health (GM070559-14) and the National Institute of Food and Agriculture, US Department of Agriculture (HATCH project 2351177000). The funders had no role in studies reported or their conclusions.

\section{Availability of data and materials}

Short-read (Illumina HiSeq 4000) sequence data are available in the NCBI SRA (accession numbers PRJNA634129). BioSample metadata are available in the NCBI BioSample database (http://www.ncbi.nlm.nih.gov/biosample/) under accession number SAMN14984385, SAMN14984386, SAMN14984388, SAMN14984390).

\section{Ethics approval and consent to participate}

The T. castaneum GA-1 strain were reared in our laboratory since 2006 from a starter culture received from USDA-ARS.

\section{Consent for publication}

Not applicable.

\section{Competing interests}

The authors declare that there are no competing interests.

Received: 2 April 2020 Accepted: 16 June 2020

Published online: 22 June 2020

\section{References}

1. Kouzarides T. Chromatin modifications and their function. Cell. 2007;128(4): 693-705.

2. Marmorstein R, Zhou MM. Writers and readers of histone acetylation: structure, mechanism, and inhibition. Cold Spring Harb Perspect Biol. 2014; 6(7):a018762.

3. Seto E, Yoshida M. Erasers of histone acetylation: the histone deacetylase enzymes. Cold Spring Harb Perspect Biol. 2014;6(4):a018713.

4. Choudhary C, Kumar C, Gnad F, Nielsen ML, Rehman M, Walther TC, Olsen JV, Mann M. Lysine acetylation targets protein complexes and co-regulates major cellular functions. Science. 2009;325(5942):834-40.

5. de Ruijter AJ, van Gennip AH, Caron HN, Kemp S, van Kuilenburg AB. Histone deacetylases (HDACs): characterization of the classical HDAC family. Biochem J. 2003;370(Pt 3):737-49.

6. Haberland M, Montgomery RL, Olson EN. The many roles of histone deacetylases in development and physiology: implications for disease and therapy. Nat Rev Genet. 2009;10(1):32-42.

7. Lagger G, O'Carroll D, Rembold M, Khier H, Tischler J, Weitzer G, Schuettengruber B, Hauser C, Brunmeir R, Jenuwein T, et al. Essential function of histone deacetylase 1 in proliferation control and CDK inhibitor repression. EMBO J. 2002;21(11):2672-81.

8. Marks PA. Histone deacetylase inhibitors: a chemical genetics approach to understanding cellular functions. Biochim Biophys Acta. 2010;1799(10-12): $717-25$.
9. Delcuve GP, Khan DH, Davie JR. Roles of histone deacetylases in epigenetic regulation: emerging paradigms from studies with inhibitors. Clin Epigenetics. 2012;4(1):5.

10. Bhaskara S, Chyla BJ, Amann JM, Knutson SK, Cortez D, Sun ZW, Hiebert SW. Deletion of histone deacetylase 3 reveals critical roles in $S$ phase progression and DNA damage control. Mol Cell. 2008;30(1):61-72.

11. Cho Y, Griswold A, Campbell C, Min KT. Individual histone deacetylases in Drosophila modulate transcription of distinct genes. Genomics. 2005;86(5): 606-17.

12. Johnson CA, Barlow AL, Turner BM. Molecular cloning of Drosophila melanogaster CDNAs that encode a novel histone deacetylase dHDAC3. Gene. 1998;221(1):127-34.

13. Foglietti C, Filocamo G, Cundari E, De Rinaldis E, Lahm A, Cortese R, Steinkuhler C. Dissecting the biological functions of Drosophila histone deacetylases by RNA interference and transcriptional profiling. J Biol Chem. 2006;281(26):17968-76.

14. Gryder BE, Wu L, Woldemichael GM, Pomella S, Quinn TR, Park PMC Cleveland A, Stanton BZ, Song Y, Rota R, et al. Chemical genomics reveals histone deacetylases are required for core regulatory transcription. Nat Commun. 2019;10(1):3004.

15. Zhu CC, Bornemann DJ, Zhitomirsky D, Miller EL, O'Connor MB, Simon JA. Drosophila histone deacetylase-3 controls imaginal disc size through suppression of apoptosis. PLoS Genet. 2008:4(2):e1000009.

16. Bodai L, Zsindely N, Gaspar R, Kristo I, Komonyi O, Boros IM. Ecdysone induced gene expression is associated with acetylation of histone $\mathrm{H} 3$ lysine 23 in Drosophila melanogaster. PLoS One. 2012;7(7):e40565.

17. Jindra M, Palli SR, Riddiford LM. The juvenile hormone signaling pathway in insect development. Annu Entomol. 2013;58:181-204.

18. D.L. Denlinger GDY, J.P. Rinehart: Hormonal control of diapause. Insect Endocrinology, Academic Press, 2012:430-463.

19. Goodman WG, and Cusson, M: The juvenile hormones. Insect Endocrinology 2012( Web.):310-365.

20. Chippendale GM, Yin CM. Reappraisal of proctodone involvement in the hormonal regulation of larval diapause. Biol Bull. 1975;149(1):151-64.

21. Riddiford LM. How does juvenile hormone control insect metamorphosis and reproduction? Gen Comp Endocr. 2012;179(3):477-84.

22. Ashok M, Turner C, Wilson TG. Insect juvenile hormone resistance gene homology with the bHLH-PAS family of transcriptional regulators. Proc Natl Acad Sci U S A. 1998;95(6):2761-6.

23. Charles JP, Iwema T, Epa VC, Takaki K, Rynes J, Jindra M. Ligand-binding properties of a juvenile hormone receptor, Methoprene-tolerant. Proc Natl Acad Sci U S A. 2011;108(52):21128-33.

24. Zhang ZL, Xu JJ, Sheng ZT, Sui YP, Palli SR. Steroid receptor co-activator is required for juvenile hormone signal transduction through a bHLH-PAS transcription factor, Methoprene tolerant. J Biol Chem. 2011;286(10):8437-47.

25. Fernandez-Nicolas $A$, Belles $X$. CREB-binding protein contributes to the regulation of endocrine and developmental pathways in insect hemimetabolan premetamorphosis. Biochim Biophys Acta. 2016;1860(3):508-15.

26. Roy A, George S, Palli SR. Multiple functions of CREB-binding protein during postembryonic development: identification of target genes. BMC Genomics. 2017;18(1):996.

27. Xu J, Roy A, Palli SR. CREB-binding protein plays key roles in juvenile hormone action in the red flour beetle, Tribolium castaneum. Sci Rep. 2018;8(1):1426.

28. Minakuchi C, Namiki T, Shinoda T. Kruppel homolog 1, an early juvenile hormone-response gene downstream of Methoprene-tolerant, mediates its anti-metamorphic action in the red flour beetle Tribolium castaneum. Dev Biol. 2009:325(2):341-50

29. Jindra M, Belles $X$, Shinoda T. Molecular basis of juvenile hormone signaling. Curr Opin Insect Sci. 2015;11:39-46.

30. George S, Gaddelapati SC, Palli SR. Histone deacetylase 1 suppresses Krüppel homolog 1 gene expression and influences juvenile hormone action in Tribolium castaneum. Proc Natl Acad Sci U S A. 2019;116(36): 17759-64.

31. Roy A, Palli SR. Epigenetic modifications acetylation and deacetylation play important roles in juvenile hormone action. BMC Genomics. 2018;19(1):934.

32. Vanhaecke T, Papeleu P, Elaut G, Rogiers V. Trichostatin A-like hydroxamate histone deacetylase inhibitors as therapeutic agents: toxicological point of view. Curr Med Chem. 2004;11(12):1629-43.

33. Grégoire S, Xiao L, Nie J, Zhang X, Xu M, Li J, Wong J, Seto E, Yang XJ. Histone deacetylase 3 interacts with and deacetylates myocyte enhancer factor 2. Mol Cell Biol. 2007;27(4):1280-95. 
34. Chuang HC, Chang CW, Chang GD, Yao TP, Chen H. Histone deacetylase 3 binds to and regulates the GCMa transcription factor. Nucleic Acids Res. 2006;34(5):1459-69.

35. Ozawa T, Mizuhara T, Arata M, Shimada M, Niimi T, Okada K, Okada Y, Ohta K. Histone deacetylases control module-specific phenotypic plasticity in beetle weapons. Proc Natl Acad Sci U S A. 2016;113(52):15042-7.

36. Chen M, Zhang N, Jiang H, Meng X, Qian K, Wang J. Molecular characterization of class I histone deacetylases and their expression in response to thermal and oxidative stresses in the red flour beetle, Tribolium castaneum. Genetica. 2019;147(3-4):281-90.

37. Urena E, Manjon C, Franch-Marro X, Martin D. Transcription factor E93 specifies adult metamorphosis in hemimetabolous and holometabolous insects. Proc Natl Acad Sci U S A. 2014;111(19):7024-9.

38. Urena E, Chafino S, Manjon C, Franch-Marro X, Martin D. The occurrence of the Holometabolous Pupal stage requires the interaction between E93, Kruppel-homolog 1 and broad-complex. PLoS Genet. 2016;12(5):e1006020.

39. Swaminathan A, Gajan A, Pile LA: Epigenetic regulation of transcription in Drosophila. Front Biosci (Landmark Ed) 2012, 17:909-937.

40. Lehrmann $\mathrm{H}$, Pritchard LL, Harel-Bellan A. Histone acetyltransferases and deacetylases in the control of cell proliferation and differentiation. Adv Cancer Res. 2002:86:41-65.

41. Li C, Liu J, Lu P, Ma S, Zhu K, Gao L, Li B, Chen K. Identification, expression and function of myosin heavy chain family genes in Tribolium castaneum. Genomics. 2019;111(4):719-28.

42. Wells $\mathrm{L}$, Edwards KA, Bernstein SI. Myosin heavy chain isoforms regulate muscle function but not myofibril assembly. EMBO J. 1996;15(17):4454-9.

43. Seebacher F, Simmonds AIM. Histone deacetylase activity mediates thermal plasticity in zebrafish (Danio rerio). Sci Rep. 2019;9(1):8216.

44. Kavaler J, Fu W, Duan H, Noll M, Posakony JW. An essential role for the Drosophila Pax2 homolog in the differentiation of adult sensory organs. Development. 1999;126(10):2261-72.

45. Duchek P, Somogyi K, Jekely G, Beccari S, Rorth P. Guidance of cell migration by the Drosophila PDGFNEGF receptor. Cell. 2001;107(1):17-26.

46. Narita N, Fujieda S, Kimura Y, Ito Y, Imoto Y, Ogi K, Takahashi N, Tanaka T, Tsuzuki H, Yamada T, et al. Suppression of histone deacetylase 3 (HDAC3) enhances apoptosis induced by paclitaxel in human maxillary cancer cells in vitro and in vivo. Biochem Biophys Res Commun. 2010;396(2):310-6.

47. Glass CK, Rosenfeld MG. The coregulator exchange in transcriptional functions of nuclear receptors. Genes Dev. 2000;14:121-41.

48. Kao HY, Downes M, Ordentlich P, Evans RM. Isolation of a novel histone deacetylase reveals that class I and class I| deacetylases promote SMRTmediated repression. Genes Dev. 2000;14(1):55-66.

49. Ishizuka T, Lazar MA. The N-CoR/histone deacetylase 3 complex is required for repression by thyroid hormone receptor. Mol Cell Biol. 2003;23(15):5122-31.

50. Pile LA, Schlag EM, Wassarman DA. The SIN3/RPD3 deacetylase complex is essential for $\mathrm{G}$ (2) phase cell cycle progression and regulation of SMRTER corepressor levels. Mol Cell Biol. 2002;22(14):4965-76.

51. Rundlett SE, Carmen AA, Kobayashi R, Bavykin S, Turner BM, Grunstein M. HDA1 and RPD3 are members of distinct yeast histone deacetylase complexes that regulate silencing and transcription. Proc Natl Acad Sci U S A. 1996;93(25):14503-8

52. Yang X, Wu Q, Zhang L, Feng L. Inhibition of histone deacetylase 3 (HDAC3) mediates ischemic preconditioning and protects cortical neurons against ischemia in rats. Front Mol Neurosci. 2016;9:131.

53. Zhang X, Wharton W, Yuan Z, Tsai SC, Olashaw N, Seto E. Activation of the growth-differentiation factor 11 gene by the histone deacetylase (HDAC) inhibitor trichostatin a and repression by HDAC3. Mol Cell Biol. 2004;24(12): 5106-18.

54. Kim HC, Choi KC, Choi HK, Kang HB, Kim MJ, Lee YH, Lee OH, Lee J, Kim YJ, Jun W, et al. HDAC3 selectively represses CREB3-mediated transcription and migration of metastatic breast cancer cells. Cell Mol Life Sci. 2010;67(20): 3499-510.

55. Carre C, Szymczak D, Pidoux J, Antoniewski C. The histone H3 acetylase $\mathrm{dGcn} 5$ is a key player in Drosophila melanogaster metamorphosis. Mol Cell Biol. 2005;25(18):8228-38.

56. Haliscak JP, Beeman RW. Status of malathion resistance in five genera of beetles infesting farm-stored corn, wheat, and oats in the United States. J Econ Entomol. 1983;76(4):717-22.

57. Goodman CL, Stanley D, Ringbauer JA Jr, Beeman RW, Silver K, Park Y. A cell line derived from the red flour beetle Tribolium castaneum (Coleoptera: Tenebrionidae). In Vitro Cell Dev Biol Anim. 2012;48(7):426-33.
58. Gaddelapati SC, Dhandapani RK, Palli SR. CREB-binding protein regulates metamorphosis and compound eye development in the yellow fever mosquito, Aedes aegypti. Biochimica et Biophysica Acta (BBA) - Gene Regulatory Mechanisms. 1863;2020(8):194576.

\section{Publisher's Note}

Springer Nature remains neutral with regard to jurisdictional claims in published maps and institutional affiliations.
Ready to submit your research? Choose BMC and benefit from:

- fast, convenient online submission

- thorough peer review by experienced researchers in your field

- rapid publication on acceptance

- support for research data, including large and complex data types

- gold Open Access which fosters wider collaboration and increased citations

- maximum visibility for your research: over $100 \mathrm{M}$ website views per year

At BMC, research is always in progress.

Learn more biomedcentral.com/submissions 